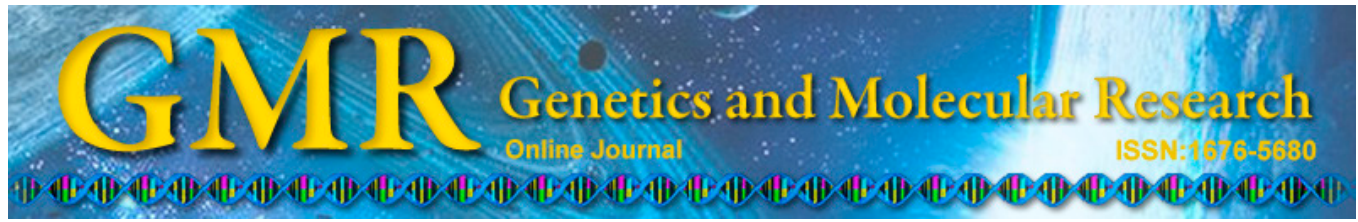

\title{
Pathology of the lower nasolacrimal duct mucosa at multiple time points after lacrimal retrograde catheterization in rabbit
}

\author{
L. Ye, Y. Peng, Y. Wang, K. Zeng and J.X. Zhang \\ Shenzhen Eye Hospital, Ji-nan University, Shenzhen, China \\ Corresponding author: L. Ye \\ E-mail: yelin_yl@yeah.net
}

Genet. Mol. Res. 14 (3): 7929-7936 (2015)

Received December 10, 2014

Accepted April 6, 2015

Published July 14, 2015

DOI http://dx.doi.org/10.4238/2015.July.14.19

\begin{abstract}
Lacrimal duct obstruction is a common eye disease, and obstruction of the nasolacrimal duct accounts for over $50 \%$ of these cases. Nasolacrimal duct obstruction is usually treated surgically. Lacrimal retrograde catheterization is a novel surgical approach with a high success rate and a relatively high recurrence rate. In this study, we examined the postoperative pathological changes in the lower nasolacrimal duct mucosa at multiple time points $(2,4,6,8,10,12$, and 14 weeks) after retrograde lacrimal dilated drainage tube implantation in rabbits. One side of the nasolacrimal duct was obstructed in 14 rabbits. Compared to the control side, the 2-, 4-, 6-, and 8-week groups presented no obvious changes in hematoxylin and eosin (H\&E)-stained tissue. The 10-week group presented isolated granulomas. The 12- and 14-week groups presented scattered granulomas. The granulomas were smaller and the density of fibroblasts was lower in the 12-week group compared with the 14 -week group. Transforming growth factor- $\beta 1$ immunohistochemistry resulted in strong immunoreactivity in the 14-week group and weak immunoreactivity in the 12-week group, and the difference was statistically significant $(\mathrm{P}<0.05)$. Fibroblast apoptosis was observed in the 4- and 6-week groups, and disorganized fibers were observed in the
\end{abstract}


10-week group. Most fibroblasts in the 12- and 14-week groups were the active type with mechanocytes. Granulomas were induced after a long time by the implantation of silicone tubes in rabbits. Thus, recurrent obstructions of the nasolacrimal duct may be caused by granulomas, progressive fibrosis, and/or adhesion of the surrounding tissues.

Key words: Retrograde lacrimal dilated drainage tube implantation; Lower segment of the nasolacrimal duct mucosa; Pathology

\section{INTRODUCTION}

Lacrimal duct obstruction is a common eye disease, and obstructions of the nasolacrimal duct account for more than $50 \%$ of these cases. Surgery is the routine treatment for nasolacrimal duct obstruction (Nemet and Vinker, 2014), and lacrimal retrograde catheterization is a novel and simple surgical approach that has a high success rate and a relatively high recurrence rate. Moreover, it is not clear how long the postoperative lacrimal drainage tubes should remain implanted. In this study, a rabbit model of nasolacrimal duct obstruction was created with mechanical friction by injecting a sclerosing agent injection and then performing lacrimal retrograde catheterization. Pathology in the lower nasolacrimal duct mucosa was examined in order to investigate the effects of the retention of nasal lacrimal drainage tubes.

\section{MATERIAL AND METHODS}

\section{Equipment and reagents}

A balloon catheter with a guide wire, a lacrimal probe with a needle core, and a ND-09A lacrimal drainage tube expansion were purchased from Hangzhou Tu-qing Pharmaceutical Co., Ltd. (Hangzhou, China) (shown in Figures 1 and 2). The sclerosing agent (gelatin sponge + compound diatrizoate injection) was produced by Guilin Norwich Company. The 846 Mixture was bought from Shengda Animal Drugs, Ltd. [Dunhua City, Jilin Province, China; No. (2010) 070031582]. The urethane was from Shanghai Heng Yuan Biotechnology Co., Ltd.

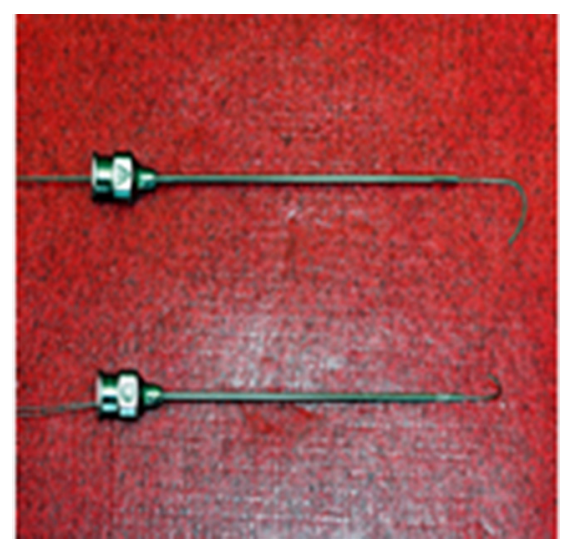

Figure 1. Lacrimal probe with needle core. 


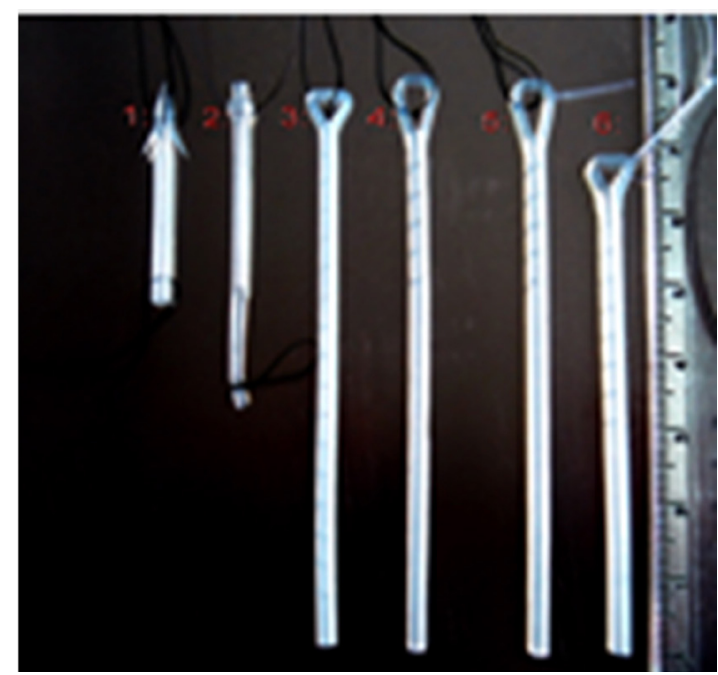

Figure 2. Dilating lacrimal duct drainage tube.

\section{Experimental animals and grouping}

Fourteen 2-month-old male and female healthy purebred New Zealand white rabbits were provided by the animal house of Peking University Shenzhen Hospital, and their weights ranged from $2.0-2.5 \mathrm{~kg}$. The rabbits were randomly divided into seven groups according to when they were killed after the surgery $(2,4,6,8,10,12$, or 14 weeks), and there were two in each group (four eyes). The left and right eyes were randomly divided into the experimental group (the eye that was surgically treated) and the control group (the other eye that was not surgically treated).

\section{Establishment of the lacrimal duct obstruction model}

After 1 week of adaptive feeding, the rabbits were grouped randomly, and their eyes were flushed to ensure that the lacrimal ducts were clear. Each rabbit was anesthetized by intraperitoneal injections with $0.2 \mathrm{~mL} / \mathrm{kg} 846$ Mixture. After sterilization of the ocular region, a balloon catheter with a guide wire $(2.0 \mathrm{~mm}$ in diameter) was used to probe into the lower segment of the nasolacrimal duct where a length of about $42 \mathrm{~mm}$ was marked. The balloon was filled to a pressure of 6-8 atm so that there was resistance when it was pulled back. It was then rubbed onto the lacrimal mucosa, and the friction length was marked. The pressure was withdrawn. After a 1 min rest, the whole process was repeated three times before the balloon catheter was removed. The lacrimal probe was put into the nasolacrimal duct in order to inject the sclerosants (gelatin sponge + compound diatrizoate injection). The lacrimal probe was withdrawn, and the rabbits continued to feed for about one week. When the symptoms of epiphora/overflow pus were exhibited, another round of lacrimal passage irrigation was performed. Liquid reflux occurred in the model eye, while the lacrimal duct in the other eye remained clear. Fourteen lacrimal duct obstruction model eyes were successfully established in 14 rabbits. 


\section{Preoperative preparation and anesthesia}

About $4 \mathrm{~mL} / \mathrm{kg} \mathrm{10 \%}$ urethane was used for intravenous anesthesia and $0.1 \%$ tetracaine hydrochloride was used for conjunctival surface anesthesia (twice). A Gentamicin injection and dexamethasone injection were done during the lacrimal irrigation to rinse the pus.

\section{Surgical methods}

The lacrimal guide pins were used for lacrimal probing, and the "9" on the needle-handle was made to point toward the anterior nasal aperture. With one hand fixing the needle, the other hand was used to convey the needle core downwards until it came out of the nasal cavity. The traction line of the lacrimal expansion drainage tube was penetrated between the doublestranded needle core, and the needle core was pulled up until it could not be pulled together with the needle and the traction line was pulled out from the lacrimal punctum. The traction line was pulled until the marker on the ND-09A tube was about $1 \mathrm{~cm}$ from the lacrimal punctum. Lacrimal irrigation was again performed, and the traction line was cut to end the surgery.

\section{Time points, items, and methods of observation}

The tubes were removed at different time points after the surgery $(2,4,6,8,10,12$, or 14 weeks), and the pathological changes in the lacrimal duct lumen were examined under a microscope.

\section{Collection of specimens}

The animals were sacrificed by the air embolism method at each time point in order to collect both sides of the nasolacrimal duct. The details of the method were as follows. Fluorescein was injected from the lacrimal punctum, and the skin was cut from the palpebral fissure to the oral fissure to the subcutaneous layer to expose the maxilla, premaxilla and part of the cheek bone. The bone was nibbled open from the port side of the maxilla so that the fluorescein staining in the nasolacrimal duct could be seen. The bone window was expanded along the nasolacrimal duct forward and backward in order to fully expose the entire duct, and the lower part was then removed.

\section{Routine pathological examination}

The tissue was fixed with $10 \%$ formalin and embedded after $24 \mathrm{~h}$ in paraffin. Slices were then made with an ultramicrotome, and they were examined under an optical microscope after hematoxylin and eosin (H\&E) staining.

\section{RESULTS}

H\&E staining of the nasolacrimal duct tissue of the control eyes showed that the epithelium was covered by squamous cells. Beneath the epithelium was the extracellular matrix that contained a large amount of elastic fibers and collagen fibers, and the vascular plexus could be seen. The muscular layer was composed of smooth muscle (Figure 3). 


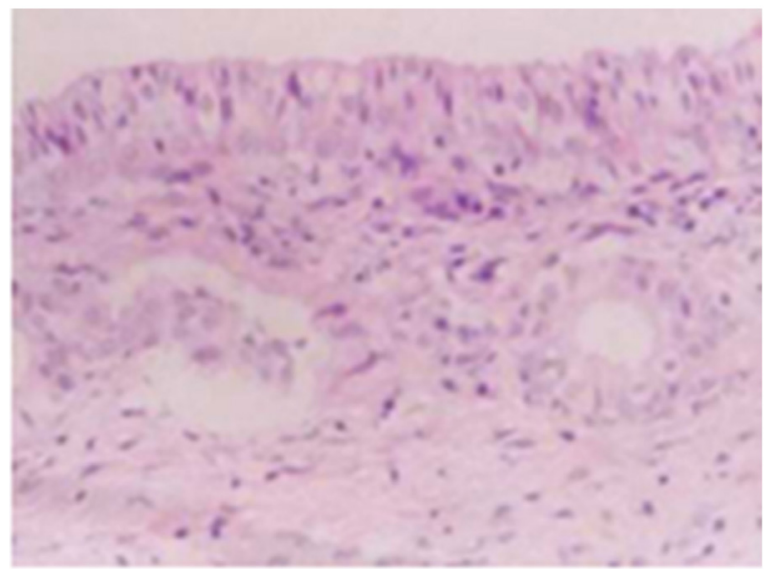

Figure 3. Nasolacrimal duct tissue of the control eye.

Compared to the tissue in control group, the tissue that was collected 2 and 4 weeks after surgery exhibited edema of the lower nasolacrimal duct and abundant inflammatory cell infiltration. In the tissue collected 6 and 8 weeks after surgery, the infiltration of inflammatory cells was decreased notably, and there was still no sign of granulomatous tissue (Figures 4 and 5). The appearance of isolated granulomatous tissue in the interstitial tissue started from 10 weeks after surgery, and the tissue that was collected at 12 and 14 weeks exhibited scattered granulomatous tissue that could be observed within the submucosal tissue and that was manifested as homogeneous and lightly stained areas that were surrounded by epithelial cells (with a large cell body, abundant cytoplasm in pale pink, and a round, oval, or spindle cucumber-like shaped nucleus and other forms, and one to two nucleoli) at the early stage. In the granulomatous tissue, there were newly formed capillaries, scattered multinucleated macrophages, and epithelial cells surrounded by proliferated fibrous connective tissue (Figure 6). H\&E staining showed that the granulomas in the 14-week tissue were larger than those in the 12-week tissue. In addition, the density of the epithelial cells and fibroblasts in the 14-week tissue was higher than that in the 12-week tissue, and they were closer to the center of the granulomas.

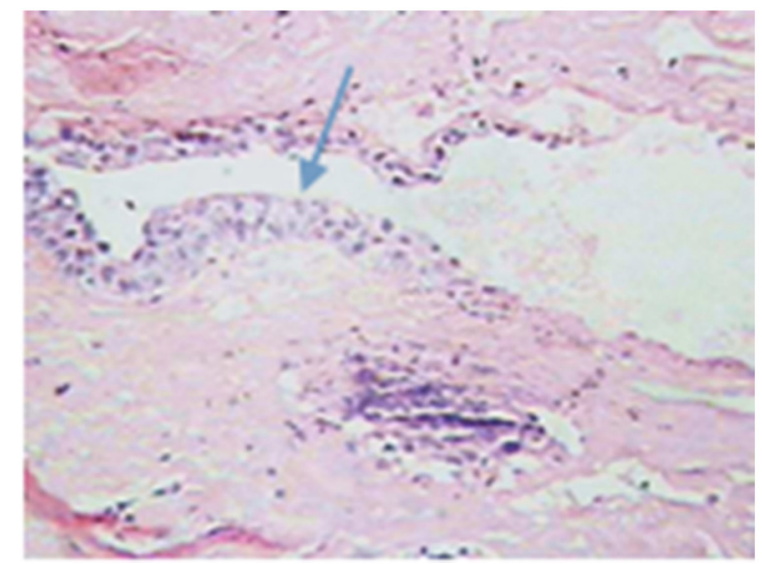

Figure 4. Two weeks after surgery, inflammatory cells were seen (indicated by the arrow). 


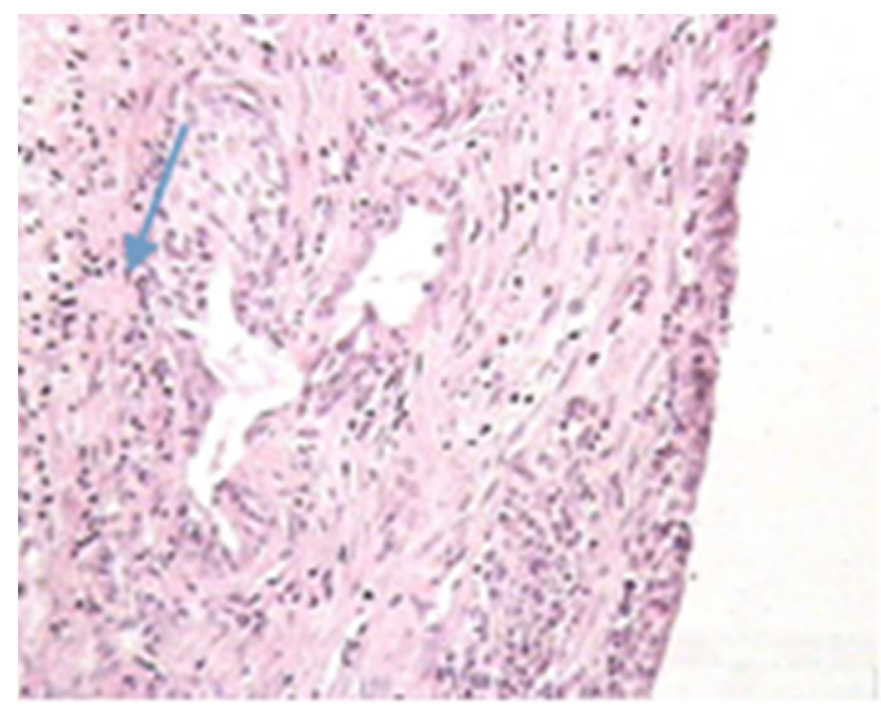

Figure 5. Four weeks after surgery, inflammatory cells were seen (indicated by the arrow).

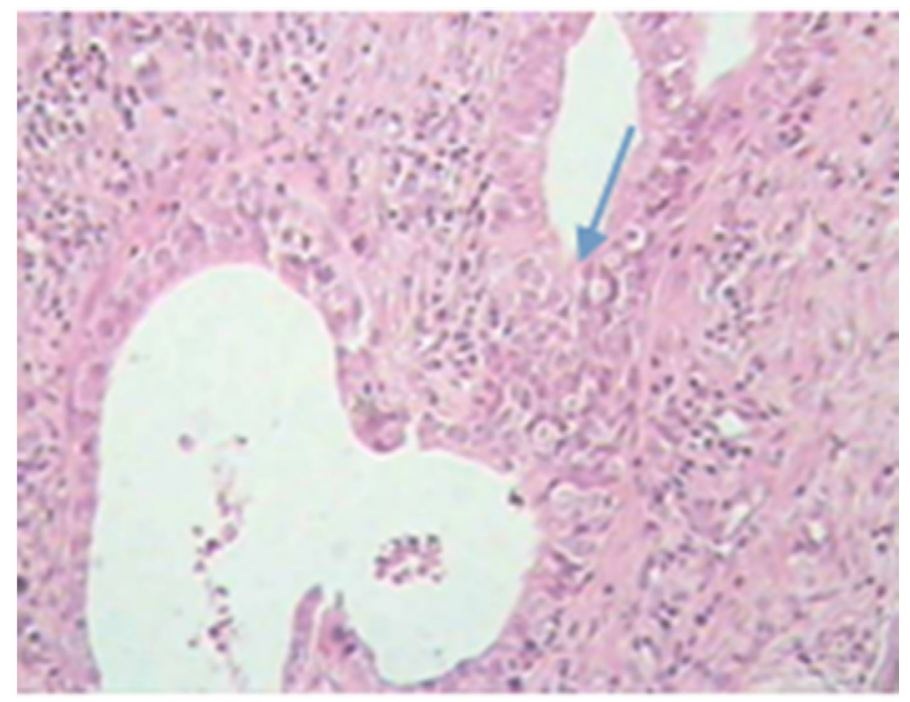

Figure 6. Twelve weeks after surgery, isolated granuloma tissue was seen.

\section{DISCUSSION}

Since the first external nasal cavity anastomosis that was performed by Toil in 1904, a dacryocystorhinostomy has been recognized as the best method for the treatment of chronic dacryocystitis. However, facial surgeries result in scars (Lei, 2004). The application of nasolacrimal duct retrograde catheterization in the treatment of chronic dacryocystitis has been 
done for the last 20 years. In recent years, the successful development of new support has made lacrimal intubation popular again (Zhang et al., 2007). The equipment used in our study was the ND-09A lacrimal expansion drainage tube that is manufactured by Hangzhou Tu Qing Pharmaceutical Co., Ltd. It is made of medical silicone with good biocompatibility, and an inverted triangle supports the lacrimal sac from within and fixes the tube. The drainage tube's large diameter and holes on the thick wall make it quite effective for dilating the nasolacrimal duct, which results in a favorable drainage effect. However, in clinical practice, some patients have experienced reoccurrence of the obstruction after removal of the tube, which may be related to the length of time that the tube is implanted (Li et al., 2009; Liu et al., 2010; Eshraghi et al., 2014). Currently, it is not clear how long the postoperative lacrimal drainage tube should remain implanted, and the basic studies that have examined this issue have been insufficient.

The nasolacrimal duct tissue of rabbits and humans is quite similar. Both are composed of two layers of cells: the epithelial and the interstitial layers. In addition, the structure of each layer of the intraosseous part is similar to that in humans, while the intranasal segment lacks the adenoid layer below the epithelium (Sun, 1996). In the present study, obstruction models that were created by mechanical friction and an injection of a sclerosing agent (Hu et al., 2004), were used to examine the pathological changes in the lower lacrimonasal duct after retrograde lacrimal intubation in order to determine the optimal withdrawal time of the tube. In our results, compared with the control group, no manifestation of granulomatous tissue was observed in the mucosa of the lower lacrimonasal duct within 10 weeks of the surgery. In contrast, the postoperative infiltration of inflammatory cells and the proliferation of fibroblasts and granulomatous tissue began to appear after 12 weeks.

The granulomatous tissue was mainly composed of epithelial cells and multinucleated giant cells, and its development, fibrosis, and adhesion to surrounding tissue very likely contributed to the restenosis and reoccurrence of the obstruction of the nasolacrimal duct. The presence of this tissue was a possible reason for the reoccurrence of the obstruction after the removal of the retrograde draining tubes. Kashkouli (2010) reported that, in severe cases and cases with re-obstruction, the amount of granulomatous tissue and fibroblasts were significantly higher compared with patients who were diagnosed with a nasolacrimal duct obstruction for the first time.

Therefore, these findings suggest view that the withdrawal time of the drainage tube should be within 12 weeks of the surgery. If not, the granulomatous tissue proliferation will likely cause a second obstruction.

\section{ACKNOWLEDGMENTS}

Research supported by the Shenzhen Science and Technology Program (\#200,902,121).

\section{REFERENCES}

Eshraghi B, Masoomian B, Izadi A, Abedinifar Z, et al. (2014). Conjunctival bacterial flora in nasolacrimal duct obstruction and its changes after successful dacryocystorhinostomy surgery. Ophthal. Plast. Reconstr. Surg. 30: 44-46.

Hu XW, Zhang XN and Bai X (2004). Experimental study of rabbit nasolacrimal duct anatomy. Huazhongkejidaxuexuebao 43: $210-212$.

Kashkouli MB, Sadeghipour A, Kaghazkanani R, Bayat A, et al. (2010). Pathogenesis of primary acquired nasolacrimal duct obstruction. Orbit 29: 11-15. 
Lei F (2004). The experience of dacryocystorhinostomy (DCR)-century memory for Toti surgery. Chin. J. Ocul. Trauma Occup. Eye Dis. 7: 450-451.

Li J, Duan ZJ, Du XG, Guo LX, et al. (2009). A clinical efficacy study of 4 treatment methods for te ar-dluct blockage. Yanwaishang zhiyebing zazhi 31: 873-876.

Liu FL, Liu HC, Zeng YP, Wang Q, et al. (2010). Clinical observation of laser assisted dacryocytoplasty combined with new Lacrimal drainage tube indwelling surgery for obstruction of lacrimal passage. Yankexinjinzhan 30: 172-174.

Nemet AY and Vinker S (2014) Associated morbidity of nasolacrimal duct Obstruction - a large community based casecontrol study. Graefes Arch. Clin. Exp. Ophthalmol. 252: 125-130.

Sun WR (1996). Editor in chief, yankebinglixue. Beijing People's Medical Publishing House, Beijing, 629-676.

Zhang JX, Xiang N and Deng HW (2008). Attention to the mini-invasive treatment of the dacryagogatresia disease. Ophthalmology 2: 79-81. 